\title{
The Effect of Acute Vibration Training on Delayed Onset Muscle Soreness in Young Non-Athlete Women
}

\author{
Raheleh Kamandani ${ }^{1,}$, , Farsahd Ghazalian ${ }^{2}$, Khosro Ebrahim ${ }^{2}$, Neda Ghassembaglou ${ }^{2}$, \\ Mehdi Shiri Piraghaj ${ }^{3}$, Alireza Khorram ${ }^{4}$ \\ 1 Department of Education and Sport Sciences, Science and Research Branch, Islamic Azad University, Tehran, IR Iran \\ 2 Department of Physical Education and Sport Sciences, Science and Research Branch, Islamic Azad University, Tehran, IR Iran \\ ${ }_{4}^{3}$ Medical Laboratory, Tahghigh Pathobiology Laboratory Center, Tehran, IR Iran \\ 4 Health Promotion Research Center, Zahedan University of Medical Sciences, Zahedan, IR Iran \\ ${ }^{*}$ Corresponding author: Raheleh Kamandani, Department of Education and Sport Sciences, Science and Research Branch, Islamic Azad University, Tehran, IR Iran. Tel/Fax: +98- \\ 2144525942, E-mail: k_raheleh@yahoo.com.
}

Received: October 29, 2012; Revised: August 28, 2013; Accepted: August 30, 2013

\begin{abstract}
Background: Delayed onset muscle soreness (DOMS) is the sensation of stiffness and pain in the muscles that occurs 24-48 hours after unaccustomed or strenuous exercise.

Objectives: The aim of this study was to examine and compare the effect of vibration training(VT) on Delayed onset muscle soreness. Materials and Methods: Twenty healthy non-athletic volunteers (Female, age 20-30 years, body mass $23.27 \pm 0.82 \mathrm{~kg}$ ) were randomly assigned to $\mathrm{VT}(\mathrm{n}=10)$ and non-VT $(\mathrm{n}=10)$ groups. A vibrator was used to apply $50-30 \mathrm{~Hz}$ of vibration on the left quadriceps and calf muscles in the VT group, while no vibration was applied for the non-VT group. Then, both groups ran downhill on a $-5^{\circ}$ treadmill at a speed of 5-7.5 km/hour (increasing speed). Twenty-four-hours before and after the vibration training, serum creatine kinase levels, pressure pain threshold and the rate of edema were measured.

Results: Compared with the non VT group, the VT group showed a significant decrease in pressure pain threshold at $15 \mathrm{~cm}$ of patellar point $(\mathrm{P}=0.042)$ and calf muscle $(\mathrm{P}=0.041)$, there were no significant differences between groups in the pressure pain threshold at $5 \mathrm{~cm}$ $(\mathrm{P}=0.509)$ and $10 \mathrm{~cm}(\mathrm{P}=0.152)$ points. There was no significant change in the edema rate at the Gastrocnemius muscle $(\mathrm{P}=0.420)$ and midpoint of quadriceps muscle $(P=0.380)$ in VT and non-VT groups. Despite the increase in creatine kinase enzymes in the two groups, there was no statistically significant difference between the groups $(\mathrm{P}=0.173)$.

Conclusions: The results showed that using the vibration training can affect delayed onset muscle soreness (DOMS) reduction between non-VT and VT groups. Further studies with larger samples are needed to determine the vibration training positive and negative effects.
\end{abstract}

Keywords: Vibration; Exercise; Creatine Kinase

\section{Background}

Delayed onset muscle soreness (DOMS) is the sensation of stiffness and pain in muscles, which occurs 24-48 hours after unaccustomed or strenuous exercise (1). In some studies, muscle damage due to eccentric exercise is associated with reduced muscle performance (2). Manifestations of DOMS are muscle damage including pain, spasm, decreased range of motion and functional strength as well as biochemical signs such as increasing creatine kinase (CK) and lactate dehydrogenase (LDH) enzymes (3); some studies have indicated that after eccentric exercise, omega-3 supplementation is a decreasing marker of soreness (4). The presence of muscle enzymes in the blood after intense exercise indicates some structural damage in the muscle cell membrane and the increase rate is 2-10 times their normal amount. Some sources have stated that $\mathrm{Z}$ lines are torn due to increased stress or pulling muscle fibers in eccentric contraction of the muscle $(5,6)$. Localized anemia leads to accumulation of an unknown pain substance that causes stimulation of nerve terminals, associated with muscle pain. Muscle pain causes reflective seizure that can lead to localized anemia (7). Finding ways to reduce the incidence and severity of muscle pain is one of the important aspects of sports and health sciences (1). Anti-inflammatory drugs, antioxidants, ultrasound, massage, stretching exercises, cryotherapy methods have been tested to reduce DOMS, while another method was vibration training before eccentric exercise. In some cases, a combination of one or more of these described methods were adapted by researchers but due to their lack of reliability and validity, there is no consensus among researchers (1). Currently, vibration training has been promoted as a new, effective and efficient training method and is widely applied in sports and rehabilitation institutes. Although there is no clear consensus on the mechanisms yet most 
studies have proposed a warming-up effect (8), increases in central and peripheral nervous system, stimulation of motor units, reduction of the threshold of the Golgi organs, prevention of antagonist muscle activity, activation of gamma motor units and muscle spindles were the physiological reason for WBV effects $(5,9,10)$. Nasarov stated that vibration increases range of motion in involved joints if a change has occurred in the pain threshold. He also assumed that vibration in addition to improvement of flexibility, improves blood flow (11). Kerschan (2001) in the study that was conducted on 20 healthy subjects which aimed to examine the effect of low frequency whole body vibration on the peripheral circulation showed that low-frequency vibration increases muscle blood flow and increases muscle strength but in previous studies that had used high frequency negative effects on muscle blood flow was observed (12). Bakhtiari and colleagues (2007) reported that maximum contraction strength in quadriceps muscle in the control group decreased in comparison to the experimental group and creatine kinase level was increased (13). Lau (2011) studied the vibration remedial effects on muscle damage due to eccentric training. Results showed that vibration training is effective in reducing delayed onset muscle soreness and recovery of range of motion after intense eccentric exercise but doesn't have an effect on the swelling and recovery of muscle strength and serum creatine kinase activity (14). Gojanovic et al. showed that whole-body vibration (WBV) increases creatine kinase levels but this increase is not harmful and the amount of this enzyme somewhat results drug induced myopathy (9). During the last decade whole-body vibration have been recommended as a training program and rehabilitation technique. Studies in this area like other previous methods involves uncertainty. In some studies, positive effects (13), some others negative effects and in some no effect have been reported (10). These differences may be due to the frequency, amplitude and duration of vibration training (15). At the beginning of the study, the hypothesis of the study was that high-frequency vibration can lead to increased blood circulation and positive effects on vibration training, so we applied high-amplitude pulses with high frequency build up.

\section{Objectives}

The main objective of this study was to examine and compare the effect of vibration training (VT) on delayed onset muscle soreness (DOMS) and prevention.

\section{Materials and Methods}

Twenty healthy non-athletic volunteers (Female, age 2030 years) participated in the study. The subjects were nonathletic young women. They were university students and employees and were living in Tehran. The research samples were assigned into VT $(\mathrm{n}=10)$ and non-VT $(\mathrm{n}=$ $10)$ groups by the simple random method. Subjects were excluded if they: (7) were pregnant, (11) had a history of neuromuscular disease, musculoskeletal disorders, advanced osteoarthritis joint disease, implants in the hip and knees and lower extremity fractures, (15) had a diagnosis of cardiovascular disease, (13) had a history of respiratory infection, (16) were elite athletes, (3) had exercise or physical activity during the last one month, (17) taking vitamin supplementation or pain medication in the last 10 days. All subjects were aware of the purpose of the study and read and signed an informed written consent form. To ensure the subjects' health status one day before the study, all participants were evaluated by an internist to confirm that they were in good physical health. Echocardiographic examination was performed for all 25 participants, but three of them were diagnosed with heart failure (mitral valve disease) and were excluded from the study. Using a random allocation method, subjects were divided into two groups; control $(\mathrm{N}=10)$ and experimental $(\mathrm{N}=10)$ groups. A pre-test and post-test following the vibration session were performed 24 hours before and after the treatment interventions. The height and weight and BMI were measured.

A vibrator was used to apply $30-50 \mathrm{~Hz}$ of vertical vibration on the left quadriceps and calf muscles in the VT group, while no vibration was applied for the non-VT group. Next, both groups ran downhill on a $-5^{\circ}$ treadmill at a speed of 5-7.5 km/hour (increasing speed). Each session took place at the Stadium of National Olympic Academy. The control group only performed the eccentric exercises; they walked downhill on $a-5^{\circ}$ treadmill to ensure the development of muscle soreness during eccentric activity and two participants (as a pilot group) performed the training before doing the study. Twenty-four hours before and after the vibration training, serum creatine kinase levels, pressure pain threshold and the rate of edema were measured.

Measured variables included:

1. Measurement of pressure pain thresholds over the Quadriceps Femoris and Gastrocnemius muscle:

Measurements were taken before and after the exercise while lying down at 5,10 and $15 \mathrm{~cm}$ and a 20-mL syringe was placed above the patella and measured pressure pain thresholds over the quadriceps muscles. There was a spring inside that scaled the muscle and we asked the patients to announce any unpleasant sensation and pain and the number on the syringe was recorded. Next, by changing the position and lying on the belly, the distance from the patella to the midpoint of the calf muscle was scaled and pressure pain thresholds were then measured. Measurements were recorded 24 hours before and after the eccentric exercise.

2. Measurement of creatine kinase levels:

The subjects in two groups (control and experimental) attended the laboratory 24 hours before and after the eccentric exercise and blood samples $(\sim 5 \mathrm{~mL})$ were taken.

3. Measurement of edema volume in the mid-thigh and mid-calf muscles: 
We measured distance from the patella to the groin, and periphery of the thigh marked by a tape measure while the participants were in standing position and the maximum dimension of gastrocnemius muscle was measured. Two measurements were taken from each subject $24 \mathrm{~h}$ before and after the eccentric exercise. The eccentric exercise protocol is illustrated by Table 1.

Table 1. Eccentric Training Protocol

\begin{tabular}{lll}
\hline Cycle & Rate, $\mathbf{k m . h}$ & Time, min \\
\hline Warm up & 5 & 10 \\
\hline Jugging & 6 & 10 \\
Running & 7 & 10 \\
Running & 7.5 & 5 \\
Cool down & 5 & 5 \\
\hline
\end{tabular}

In the VT group, eccentric exercise was performed after vibration training. This study was an incremental training protocol with constant amplitude and frequency with incremental power and measurement of its effect on subjects that has not been seen in other studies. Evaluation of the comparative effect of vibration training performed pre- and post-eccentric exercise at different time periods. We applied high-amplitude pulses with high frequency build up.

Vertical vibration exercise protocol was as follows:

The VT group received vertical vibration loading on a WBV platform in a standing position for 3 minutes. Next, both groups, the VT and non-VT groups performed 5 sets of eccentric training, separated by $30 \mathrm{sec}$ intervals, to induce DOMS and they were allowed to have a 30-sec rest between each condition (Table 2).

Table 2. Vibration Training

\begin{tabular}{|c|c|c|c|c|c|}
\hline Position & Amplitude, mm & Frequency, Hz & Duration of Training, $s$ & Set & Rest Duration, s \\
\hline Semi squat & 12 & 30 & 30 & 1 & 30 \\
\hline Semi squat & 12 & 35 & 45 & 1 & 30 \\
\hline Semi squat & 12 & 40 & 60 & 1 & 30 \\
\hline Semi squat & 12 & 45 & 45 & 1 & 30 \\
\hline Semi squat & 12 & 50 & 30 & 1 & 30 \\
\hline
\end{tabular}

Measurements were taken 24 hours before and after exercise and changes in measures were compared between the VT and non-VT groups. During the study, pressure pain thresholds were asked and recorded at every five minute interval, baseline Borg's perceived exertion and pain scales and at the end of study, the subjects reported numbers 17 or 18 (very hard) (18). The devices used in the study included, whole-body vibration machine-model, jet-vibe, high quality height and weight measuring instruments, stopwatch (citizen, Japan), treadmill; techno gym model and whole body vibration training was carried out using the jet-vibe system. Descriptive statistics (mean and standard deviation) were used to summarize the demographic characteristics of subjects. Covariance and multivariate analysis of variance was performed to examine the hypothesis of the study. All statistical analyses were performed using the SPSS software (ver- sion 17). Statistical significance was set at $\mathrm{P}<0.5$ for all tests.

\section{Results}

All participants were young women $(\mathrm{N}=20)$ between ages twenty and thirty (Table 3 ).

Compared with the Non VT group, the VT group showed a significant decrease in pressure pain threshold in $15 \mathrm{~cm}$ of patellar point $(\mathrm{P}=0.042)$ and calf muscle $(\mathrm{P}=0.041)$; there were no significant differences between groups regarding the pressure pain threshold at $5 \mathrm{~cm}(\mathrm{P}=0.509)$ and $10 \mathrm{~cm}$ $(P=0.152)$ points. There was no significant change in the edema rate at the Gastrocnemius muscle $(\mathrm{P}=0.420)$ and midpoint of quadriceps muscle $(\mathrm{P}=0.380)$ in VT and nonVT groups. Despite the increase of creatine kinase enzymes in two groups, there was no statistically significant difference between the groups $(\mathrm{P}=0.173)$ (Table 4$)$.

Table 3. Participants' Characteristics, Mean \pm SEM

\begin{tabular}{llllll}
\hline & $\mathbf{N}$ & Age, $\mathbf{Y}$ & Height, cm & Weight, kg & BMI, kg / m ${ }^{2}$ \\
\hline Experimental group & 10 & $25.7 \pm 2.11$ & $163.0 \pm 0.04$ & $62.3 \pm 3.60$ & $23.4 \pm 0.68$ \\
Control group & 10 & $25.3 \pm 2.70$ & $162.0 \pm 0.03$ & $61.5 \pm 4.60$ & $23.1 \pm 0.96$ \\
\hline
\end{tabular}

\section{Discussion}

Two recent studies include, Matthew R. rheain 2009 in which 16 cases were studied and the Broadbent study, which was conducted in 2010 with 29 cases. Because of fi- nancial constraints a small sample size of 25 participants were selected. At the base line of the study, 3 subjects were excluded and the study continued with 22 subjects 
Kamandani R et al.

(2 pilot and 20 participants) that were randomly divided into control and experimental groups.

Table 4. Changes in Creatine Kinase, Pressure Pain Threshold, Edema (Calf and Quadriceps) 24-Hour Before and After Whole-Body Vibration Training in VT $(\mathrm{n}=10)$ and Non-VT Groups $(\mathrm{n}=10)$

\begin{tabular}{llllll}
\hline & \multicolumn{2}{c}{ Experimental Group $(\mathbf{M} \pm \mathbf{S D})$} & \multicolumn{2}{c}{ Control Group $(\mathbf{M} \pm \mathbf{S D})$} & P value \\
\hline & Pre-Test & Post-Test & Pre-Test & Post-Test \\
\hline Creatine kinase, $\mathbf{u} / \mathbf{l}$ & $56.0 \pm 3.48$ & $74.1 \pm 3.50$ & $62.1 \pm 3.94$ & $84.7 \pm 3.83$ & 0.173 \\
\hline Pain threshold of $\mathbf{5} \mathbf{~ c m}$ of patella, $\mathbf{~} \mathbf{L}$ & $12.1 \pm 2.07$ & $12.5 \pm 2.50$ & $11.0 \pm 1.41$ & $13.1 \pm 1.28$ & 0.509 \\
\hline Pain threshold of $\mathbf{1 0} \mathbf{~ c m}$ of patella, $\mathbf{~ m L}$ & $11.6 \pm 2.17$ & $13.0 \pm 2.16$ & $12.8 \pm 1.81$ & $14.5 \pm 2.32$ & 0.152 \\
\hline Pain threshold of $\mathbf{1 5} \mathbf{~ c m}$ of patella, $\mathbf{~ m L}$ & $11.7 \pm 1.70$ & $12.6 \pm 1.89$ & $12.5 \pm 2.41$ & $14.6 \pm 2.17$ & 0.042 \\
Pain threshold in Calf muscle, $\mathbf{~} \mathbf{L}$ & $11.8 \pm 2.04$ & $12.4 \pm 1.88$ & $12.7 \pm 3.94$ & $14.6 \pm 3.85$ & 0.041 \\
\hline Mid point of Calf muscle, $\mathbf{c m}$ & $37.3 \pm 2.44$ & $37.4 \pm 2.63$ & $36.2 \pm 2.00$ & $36.3 \pm 2.27$ & 0.420 \\
\hline Mid point of quadriceps muscle, $\mathbf{c m}$ & $52.3 \pm 16.65$ & $52.3 \pm 14.57$ & $50.7 \pm 20.80$ & $50.8 \pm 18.57$ & 0.380 \\
\hline
\end{tabular}

We defined a group of non-athlete female whom during the week of the study did not have activity apart from daily physical activity. Evidence shows that a number of novice athletes and those who had recently taken up the sport, after feeling discomfort or pain caused by exercise and physical activity, may have abandoned the exercise depending on the type of exercise damage; thus the study was carried out on non-athletic women to find a way to reduce their DOMS. This study was initiated to determine the effect of vibration training with high-frequency on muscle damage protection due to activation of the motor units and distribution of muscle fiber stress and increased peripheral blood flow. This study considered both the negative and positive impacts, but emphasized on the positive effects, so we applied high-amplitude pulses with high frequency build up, which was different from many studies, however further studies are required to prove the effects of vibration training. Performing eccentric exercise causes DOMS and pain at the quadriceps muscles during activity and movement. After training the amount of pain and soreness increased in both groups. The amount of pain and soreness at the point of $15 \mathrm{~cm}$ from the patella and calf muscles in the control group was higher than the experimental group. There was no significant difference between the two groups in the amount of pain and soreness in the posttest at 10 and $5 \mathrm{~cm}$ points. Hypothesis testing and results showed that there was no significant difference in the rate of edema at calf and quadriceps muscles. Eccentric exercise increased the level of CK enzymes in blood serum of subjects and 24 hours after the training, the level of CK enzyme in VT and non-VT groups increased and the rate of this increase in the Non-VT group was more than the VT group, but there was no statistically significant difference between the two groups. In this study, the eccentric contractions led to DOMS and this manifested in both groups. The answer to the question of how eccentric contractions cause pain and soreness is that during the eccentric contraction the produced force is almost doubled that of the static contraction but the total number of cross-bridges is about 10\% higher than the static contraction (5). So this stress leads to rupture of Z lines, damage to micro-tubules and intracellular microfilaments and release of photolytic enzymes. Based on the ruptured tissues theory, muscular enzymes in the blood after intense exercise increases the rate by 2 to 10 times their normal rate and because vibration training causes less damage to the muscles, the results show decrease in the level of muscle enzymes (such as Creatine kinase), and this study showed a reduction in CK enzyme in the experimental group and there was no significant decrease between the experimental and control groups. The reasons can be: (7) the type of eccentric exercise; the lack of sufficient severity and downhill angle of treadmill can lead to a decrease in the rate of CK (11). In this study we used vibration training with high amplitude that is an increasing factor that level of Ck enzyme. So in the Kerschan study (2001), the results on 20 healthy subjects showed that low-frequency vibration can lead to blood flow increase in the muscle and can increase the strength of muscle. However in previous studies, they used a high frequency method and this method had negative effects on muscle blood flow $(12,15)$. The research was carried out during the cold season and this can cause and change the rate of creatine kinase. The differences in the results can be caused by different types of vibration training and intensity, intensity of muscle contractions, muscles involved in the exercise, age, sex and athletic background of participants. Also, the small number of participants per group due to the lack of resources can affect the generalizability of the study results and it is not generalizable for the community. Therefore, to detect significant differences with statistical power, new studies with larger populations are needed. Also, vibration exercise by increasing the peripheral blood flow can decrease pain and spasms in muscles. Generally, repeated and prolonged muscle contraction may decrease the blood flow in muscles and this reduction can lead to pain and DOMS. Vibration training can reduce spasm and pain due to the increase in the muscles blood flow. As 
Professor Nasarov has demonstrated, vibration training in addition to improving the muscles flexibility can treat blood flow (11). In this study, vibration training led to a decrease in pain and muscles' spasms in the experimental group but this reduction was not statistically significant. The results of these researches are consistent with the results of Edge and Lau studies $(14,19)$. In this study, the small effect of vibration training on DOMS treatment was the result of using amplitude and high frequency. Also, performing maximal muscular exercise without optimal preparation for exercise can accumulate metabolites in muscle due to influx of interstitial fluid by osmosis into muscle cells, which leads to edema and pain. In this study, edema and pain was observed in both groups. There is a different opinion about the effect of vibration training. Some studies considered that after eccentric contraction, the vibration training is effective in reducing the DOMS $(1,14,16,20)$. Some studies have reported that vibration training has a negative effect on muscle soreness. So, the results of the Gojanovic et al. study showed that whole body vibration increases the level of creatine kinase. They assumed that muscular contractions due to whole body vibration (WBV) can lead to muscle damage, which can be identified through the measurement of muscle enzyme levels (9). However, eccentric exercise is associated with increased creatine kinase in the blood, but in some studies, there was no change. These studies showed nonsignificant increase in creatine kinase(CK)enzyme, so this should be further investigated. Similarly, the Lau study reported that $W B V$ intervention did not affect edema rate and level creatine kinase (14). Therefore, the limitations of the present study were that the study was performed in a small population with lack of daily activities control, non-control to reduce the participants 'stress and anxiety at the time of sampling with individual differences in girls' physical activity. One uncontrolled limitation of the study was the daily activities of the participants. Using two participants as a pilot group was not enough to assess the validity and potential of the study.

We investigated the effects of VT on delayed onset muscle soreness. Based on these results, vertical vibration of 30-50 Hz was applied on the left quadriceps and calf muscles only in the VT group. Next, both groups ran downhill on a -5 treadmill at a speed of 5-7.5 km/hour. Twenty-four hours before and after the vibration training, serum creatine kinase levels, pressure pain threshold and the rate of edema was measured. The results showed that there was no significant difference in the rate of edema at calf and quadriceps muscles. CK enzyme levels increased in the two groups due to using different types of vibration training and intensity and incremental frequency, so, we couldn't investigate the positive effect of vibration training on delayed onset muscle soreness treatment. The author suggests doing this study with better research resources and larger populations to investigate the real results and also involving male athletes and professional athletes of all age groups.

\section{Acknowledgements}

There is no acknowledgements.

\section{Authors' Contribution}

Dr. Farshad Ghazalian developed the original idea and the protocol and edited the manuscript.

\section{Financial Disclosure}

There is no conflict of interest.

\section{Funding/Support}

There is no support for this study.

\section{References}

1. Rhea MR, Bunker D, Marin PJ, Lunt K. Effect of iTonic whole-body vibration on delayed-onset muscle soreness among untrained individuals.J Strength Cond Res. 2009;23(6):1677-82.

2. Olsen O, Sjohaug M, van Beekvelt M, Mork PJ. The effect of warmup and cool-down exercise on delayed onset muscle soreness in the quadriceps muscle: a randomized controlled trial. J Hum Kinet. 2012;35:59-68.

3. Damirchi A, Rahmaninia F, Biniaz A. compreitive effect dynamic and static stretch training on delayed onset muscle soreness. J Movment. 2010;4:119-35.

4. Rajabi A, Lotfi N, Abdolmaleki A, Rashid-Amiri S. The Effects of Omega-3 Intake on Delayed Onset Muscle Sorness in Non-Athlet Men. Pedagogika, psihologia ta mediko-biologicni problemi fizicnogo vihovanna i sportu. 2013;1.

5. Faulkner JA, Brooks SV, Opiteck JA. Injury to skeletal muscle fibers during contractions: conditions of occurrence and prevention. Phys Ther. 1993;73(12):911-21.

6. Pyne DB. Exercise-induced muscle damage and inflammation:a review. Aust J Sci Med Sport. 1994;26(3-4):49-58.

7. Advard F, Donald M. Exercise Physiology Text Book.; 1990.

8. Wirth B, Zurfluh S, Muller R. Acute effects of whole-body vibration on trunk muscles in young healthy adults. J Electromyogr Kinesiol. 2011;21(3):450-7.

9. Gojanovic B, Feihl F, Liaudet L, Gremion G, Waeber B. Wholebody vibration training elevates creatine kinase levels in sedentary subjects. Swiss Med Wkly. 2011;141:w13222.

10. Hopkins JT, Fredericks D, Guyon PW, Parker S, Gage M, Feland JB, et al. Whole body vibration does not potentiate the stretch reflex. Int J Sports Med. 2009;30(2):124-9.

11. Albasini A, Krause M, Rembitzki IV, Hinman MR. Using Whole Body Vibration in Physical Therapy and Sport: Clinical Practice and Treatment Exercises.: Churchill Livingstone/Elsevier; 2010.

12. Kerschan-Schindl K, Grampp S, Henk C, Resch H, Preisinger E, Fialka-Moser V, et al. Whole-body vibration exercise leads to alterations in muscle blood volume. Clin Physiol. 2001;21(3):377-82.

13. Bakhtiary AH, Safavi-Farokhi Z, Aminian-Far A. Influence of vibration on delayed onset of muscle soreness following eccentric exercise. Br J Sports Med. 2007;41(3):145-8.

14. Lau WY, Nosaka K. Effect of vibration treatment on symptoms associated with eccentric exercise-induced muscle damage. Am JPhys Med Rehabil. 2011;90(8):648-57.

15. Aminian-Far A, Hadian MR, Olyaei G, Talebian S, Bakhtiary AH. Whole-body vibration and the prevention and treatment of delayed-onset muscle soreness. J Athl Train. 2011;46(1):43-9.

16. Broadbent S, Rousseau JJ, Thorp RM, Choate SL, Jackson FS, Rowlands DS. Vibration therapy reduces plasma IL6 and muscle soreness after downhill running. $\mathrm{Br} J$ Sports Med. 2010;44(12):888-94. 


\section{Kamandani R et al.}

17. Cochrane DJ, Legg SJ, Hooker MJ. The short-term effect of wholebody vibration training on vertical jump, sprint, and agility performance. J Strength Cond Res. 2004;18(4):828-32.

18. Borg G. Borg's Perceived Exertion and Pain Scales.: Human Kinetics; 1998 .

19. Edge J, Mundel T, Weir K, Cochrane DJ. The effects of acute whole body vibration as a recovery modality following high-intensity interval training in well-trained, middle-aged runners. Eur $J$ Appl Physiol. 2009;105(3):421-8.

20. Pinto NS, Monteiro MB, Arthur AP, Paiva DN, Meyer PF, SantosFilho SD, et al. Effectiveness of a protocol involving acute wholebody vibration exercises in an adult and health individual with delayed-onset muscle soreness observed after running: a case report. Journal of Medicine and Medical Sciences. 2011;2(1):612-17. 